\title{
DEHESAS DE SIERRA MORENA Y POLÍTICAS AGROAMBIENTALES COMUNITARIAS
}

\author{
POR \\ JUAN F. OJEDA RIVERA y ROCÍO SILVA PÉREZ
}

Las dehesas: Encuadre conceptual y localización geográfica

El término dehesa, que etimológicamente proviene del vocablo latino defesa-igual a tierra defendida-, no aparece como tal hasta el siglo $\mathrm{x}, \mathrm{y}$ lo hace para designar a aquellas tierras destinadas al pastoreo, sustraidas por prerrogativa real de los derechos colectivos y concedidas a las órdenes militares y religiosas o, lo que fue más común, transformadoras en bienes concejiles. La protección real garantizaba la integridad física de estos espacios contra los posibles usurpadores y evitaba el acceso de los ganados trashumantes asociados a la Mesta, de ahí su carácter de territorio defendido.

Con el paso del tiempo -tras la crisis de la ganadería trashumante y la privatización de los terrenos de propios a raíz del proceso desamortizador del siglo XIX- el témino dehesa pierde definitivamente su acepción original y pasa a designar un sistema de explotación con unas características y ubicación geográfica muy precisas. J. J. Ibáñez entiende por dehesa «una zona de pastos y arbolados con encinas, al-

Juan F. Ojeda Rivera y Rocío Silva Pérez. Departamento Geografía Física y Análisis Geográfico Regional. Universidad de Sevilla. 
cornoques, quejigos y rebollos que forman una unidad de explotación agropecuaria ampliamente diversificada, con un alto grado de estabilidad, adaptada a la explotación de medios físicos hostiles para cualquier otra altemativa de uso, característica de áreas subdesarrolladas, capaces de mantener una escasa población por hectárea» (Ibáñez, J. J., 1988). Por su parte, para Gómez Gutiérrez «el monte o bosque adehesado es un sistema agrario o una forma de explotación de los recursos agrobiológicos característica de la banda Oeste de España limítrofe con Portugal cuyos factores detemminantes son: a) un clima semiárido o subhúmedo fuertemente fluctuante, tanto a nivel estacional como anual y b) una roca madre pobre en bases que da lugar a la formación de suelos de escasa potencia, ácidos y oligotróficos, y con predominio de la fracción arenosa» (Gómez Gutiérrez, J. M., 1987).

Ambos autores identifican, pues, a las dehesas con una zona arbolada caracterizada por la complementariedad de los usos agricolas, ganaderos $\mathrm{y}$ forestales $\mathrm{y}$, de una $\mathrm{u}$ otra forma, las circunscriben al área de Sierra Morena y a la penillanura extremeña y salmantina.

Pero además de un sistema de explotación, la dehesa constituye una unidad paisajística de gran valor natural y cultural que, a su vez, responde a una forma de propiedad grande o latifundio serrano ya que «los campos adehesados rinden poco, de ahí que para conseguir que el sistema funcione en plenitud y para obtener una producción que la haga rentable [...] se requiere una gran superficie (Valle Buenestado, 1995).

En definitiva, el objeto de este análisis (la dehesa) puede definirse como una típica unidad paisajística, que responde a una forma específica de gran propiedad y a unos mecanismos de explotación extensivos y diversificados; como un agrosistema que resulta de la adaptación sabia y secular de unas sociedades a un medio natural relativamente hostil para la producción agraria.

El agrosistema dehesa se extiende en Andalucía por 780.000 ha aproximadamente, localizadas fundamentalmente en Sierra Morena, que es el reborde o escalón meridional de la Meseta Herciniana roto y rejuvenecido por el plegamiento alpino. Las formas de Sierra Morena son, pues, redondeadas y aplanadas por la erosión de los afluentes del Guadalquivir, que han ido produciendo su individualización en comarcas o subunidades: Lomas de Ubeda, Pedroches, Sierra Norte de 
Sevilla, Sierra de Aracena... Altitudinalmente se sitúa entre los 400 y 1.000 metros, presentándose como un sistema montañoso expuesto al Sur, o sea, una gran solana que, además, recibe las lluvias atlánticas a través de la apertura oceánica de la depresión bética. A pesar de lo anterior, sus alturas y la posición continental de sus umbrías y sus espacios más orientales y septentrionales no le evitan los riesgos de heladas. Se trata de una típica montaña media mediterránea con dominio climácico del bosque de quercineas perennifolias (encinas y alcornoques) y consecuente vegetación de maquis, que sólo ha llegado a ser cultivada en una cuarta parte de su extensión, siendo sus aprovechamientos de carácter extensivo.

La adversidad del medio físico no supone tradicionalmente una limitación para el asentamiento de la población. El hombre de Sierra Morena sabe adaptarse a las condiciones del medio, no apto para la producción agrícola masiva, respondiendo a unas estrategias básicas con objetivos claros (Parejo Delgado, C., 1995): Ordenación espacial de los usos, para que cada comunidad vegetal o animal quede asociada a sus específicas condiciones ambientales y no se rompa el frágil equilibrio edáfico. (Movilidad temporal, para utilizar en cada momento o estación los recursos que proporciona cada lugar. Complementariedad de usos, para poder diversificar los aprovechamientos en una economía poco abierta. Aprovechamiento integral de las producciones primarias, para evitar el despilfarro.

Todo ello da lugar a una diversidad paisajística vinculada, a su vez, a las solanas y umbrías, a la presencia de fuentes o a la distancia de los núcleos habitados y enmarcada en el autoabastecimiento, de tal forma que en los paisajes tradicionales de cada municipio de Sierra Morena podrían encontrarse siempre -ordenados desde el espacio urbano hacia el exterior- los siguientes elementos: pequeñas huertas de regado; exiguas hazas de cultivos anuales; parcelas de olivares y viñedos; dehesas; bosques de uso común.

Esta organización multiforme y sistematizada de los espacios otorga a dichas montañas andaluzas un importante papel en las economías relativamente cerradas del Antiguo Régimen, ya que aquellos campos no sólo se constituyen en despensas autoabastecedoras de sus respectivas poblaciones, sino que, además, abastecen de frutas, carne, madera, pieles, miel... a las poblaciones de los llanos o campiñas. Estos serranos llegan a soportar mejor las crisis de subsistencia 
que los agricultores campiñeses, ya que su sabio dominio de la biodiversidad les garantiza una mayor esperanza de vida que la de sus vecinos cultivadores.

Las dehesas mariánicas no ocupan, pues, todo el espacio serrano, pero constituyen sin duda su agrosistema más característico, de tal forma que las relaciones económicas y sociales que se establecen en torno a estas explotaciones agro-silvo-pastoriles marcan la peculiar configuración de estas sierras andaluzas.

\section{Las dehesas de Sierra Morena y su proceso de marginalizacion}

Las dehesas tradicionales.-La ganadería constituye el principal elemento productivo en la explotación tradicional de la dehesa. Se trata de una ganadería muy diversificada que tenía como especies dominantes al porcino de raza ibérica y al ovino de raza merina. La rusticidad y adaptación al medio de estos animales permitía obtener el máximo aprovechamiento de los recursos disponibles: así por ejemplo, la reproducción de ambas especies estaba sujeta a un ajustado y sabio calendario que aplazaba las parideras en función del estado de los pastos y de la disponibilidad de bellota. Pero la función del ganado en las dehesas tradicionales sobrepasaba ampliamente esta óptica productiva. Su sola presencia garantizaba la regeneración de los pastos, gracias al aporte de abono orgánico y al control del matorral por medio del pastoreo. Por otra parte, la pervivencia de tan peculiar organización ganadera se sustentaba -al igual que los restantes componentes del sistema- en la presencia de una mano de obra abundante y barata. Dos oficios fundamentales eran el de pastor, dedicado al cuidado de las ovejas, y el de porquero, que se ocupaba de varear las encinas para facilitar al cerdo ibérico el engorde durante la montanera.

En cuanto a su aprovechamiento forestal, no puede olvidarse que el origen de la dehesa es un ahuecamiento del bosque de quercineas, estando constituida su masa de montes por una mezcla variable de especies arbóreas y matorrales, que se combinan en función de sucesivos procesos de tala, carboneo, pastoreo, etc. En su estado natural, las quercineas presentan un porte más arbustivo que arbóreo, por lo que para ser aprovechadas por el ganado deben ser sometidas a va-

$$
-206-
$$


rias podas, que ofrecen productos energéticos básicos (madera y leña, corcho, bellota...).

Además de la poda de los árboles, una práctica necesaria para la conservación de la dehesa es la lucha contra el matorral, cuyo avance impide el desarrollo de pastos y el acceso del ganado. La forma más generalizada de combatir el matorral en las dehesas tradicionales consistía en el cultivo de las tierras pastadas a intervalos más o menos largos, según la fertilidad de los suelos. Para ello, los pequeños propietarios y campesinos sin tierra -denominados pegujalerosarrendaban a los grandes propietarios una parte de sus explotaciones para cultivarlas de cereal por un periodo generalmente de dos años, al cabo de los cuales tenían que devolver el pegujal. Con ello, el propietario se garantizaba durante varios años el buen estado de los pastos sin coste alguno, hasta que retomaban las malas hierbas, y entonces se volvía a acudir a pegujaleros.

A este variado aprovechamiento productivo -bellota, leñas, carboneo, corcho (en el caso de las dehesas de alcornoques)...- hay que unir la importante función medioambiental desempeñada por esta masa arbórea: regulación de los recursos hídricos y mejora de la fertilidad del suelo.

Por último, el espacio agrícola -ocupado por un cultivo cerealista de largas rotaciones tenía una menor significación en las dehesas tradicionales; pero, según se ha visto, cumplía un papel de primer orden en la regeneración de los pastos y en la alimentación del ganado, para el que durante determinadas épocas del año el aprovechamiento de las rastrojeras constituía uno de los pocos recursos alimenticios disponibles.

Como forma de propiedad, la dehesa se asocia a grandes fincas $\mathrm{y}$, consecuentemente, al régimen latifundista, típico y tópico del sur y oeste de la Peninsula Ibérica. Ello debe relacionarse con el alto grado de extensividad de sus sistemas agroganaderos y con las condiciones históricas de acceso a la propiedad en estas regiones. Pero, de hecho, la perpetuación del agrosistema sólo fue posible por la peculiar configuración de la estructura de la propiedad: sin la gran mayoría de explotaciones minifundistas ubicadas en los aledaños de los pueblos, cuya inviabilidad económica empujaba a sus propietarios a emplearse en las grandes explotaciones adhesadas (en muchos casos como aparceros), tampoco podría explicarse el funcionamiento de las dehesas tradicionales. 
Pero no hemos de entender que la dehesa constituya un agrosistema inmóvil. Sus distintos elementos se han ido interrelacionando de manera flexible en el espacio y en el tiempo en función de las potencialidades edáficas, las capacidades técnicas, las coyunturas económico-sociales y los distintos destinos finalistas de sus producciones. Así, por ejemplo, en la comarca de Los Pedroches (Sierra Morena cordobesa) llegan a distinguirse «dehesas viejas», surgidas a raíz de las repoblaciones castellanas del área y su proceso de señorialización (siglos XV-XVII), «dehesas nuevas», que aparecen tras la desamortización civil de mediados del xIx. Paisajísticamente, las dehesas viejas se reconocen por la escasa densidad de arbolado y su envejecimiento, por la superficie que dentro de ellas se dedica al cereal y por la omnipresencia del ganado ovino. Las dehesas nuevas, por su parte, se reconocen por la juventud y densidad de arbolado, por estar cercadas, por su dedicación ganadera y por la coexistencia en ellas de cabañas porcinas, ovinas y bovinas (Valle Buenestado, 1985).

A pesar de dichas transformaciones, la dehesa ha sobrevivido como agrosistema completo mientras se han mantenido los principios básicos de su equilibrio interno, ya que la gestión de sus recursos requiere un tratamiento más complejo y variado que el del estricto cálculo económico de los beneficios directos obtenidos de sus productos. El medio natural pobre impone limites muy claros a las actividades puramente extractivas, por lo que cualquier intensificación de sus aprovechamientos pone en peligro su equilibrio como agrosistema.

Marginalización del sistema y respuestas ante la crisis.Lo anterior explica que desde los años sesenta se esté asistiendo a un progresivo deterioro de la dehesa, cuyo origen hay que enmarcar en el desarrollismo lineal y mecanicista que define dicha década. Los mecanismos desarrollistas van a conducir inexorablemente a la despoblación de los campos serranos y a la consecuente crisis de sus aprovechamientos tradicionales, basados, como se ha visto, en la abundancia de mano de obra y en la explotación sincrónica de diferentes recursos en unos tiempos largos. Asi, el marco económico anterior va dando lugar a unas mutaciones de los elementos configuradores de la dehesa, quebrando su funcionalidad como agrosistema. 
El proceso emigratorio privó a las dehesas de uno de sus soportes fundamentales: la existencia de una mano de obra abundante que con su trabajo garantizaba su funcionamiento. Asimismo, llevó a la desaparición de determinados oficios -podadores, porqueros, pastores, pegujaleros, carboneros- que estaban en la base de su peculiar articulación.

La «huida» de la población provocó, a su vez, el abandono de las áreas de cultivo, que pronto quedarian invadidas por el matorral impidiendo la regeneración de los pastos y el acceso del ganado. Paralelamente, el incremento salarial condujo a un descuido de la vegetación arbórea (labores de poda y tala), y su consiguiente deterioro -recuperación del porte arbustivo- llevó a una degeneración de la estructura paisajística y a una crisis de la montanera por la merma en la producción de bellotas.

Aquel éxodo rural llevó asimismo aparejado una desaparición generalizada de las poqueñas explotaciones, que serían adquiridas por los grandes propietarios. Como consecuencia de ello, se produjo una mayor concentración de la propiedad de la tierra, acompañada en muchos casos de una cierta recolonización de este espacio, con la llegada de nuevos propietarios que veían en la adquisición de las dehesas un símbolo de prestigio social (Casa de Velázquez, 1986) ${ }^{1}$.

En ganadería, los cambios más importantes vienen marcados por las modificaciones experimentadas por la cabaña y por las alteraciones introducidas en el sistema de explotación. En lo que se refiere al primer punto, cabe destacar un avance espectacular de la especie bovina (preferida frente al ovino por su fácil manejo en un contexto caracterizado por la escasez de pastores) y un retroceso variable del resto de las especies y, en particular, del porcino, cuyos efectivos se vieron aminorados por el propio abandono de la dehesa, por la com-

1 Entre 1962 y 1982 desaparecieron de Sierra Morena cerca de 15.000 explotaciones que suponen más del $30 \%$ de las existentes en la primera fecha. La consecuencia ha sido una mayor concentración de la estructura de la propiedad. Así, si en 1962 las explotaciones de más de 500 has ocupaban el 43\% de la superficie mariánica, en 1989 la proporción de explotaciones con esta dimensión oscila entre el $70 \%$ y el $80 \%$, según comarcas. Por otra parte, los resultados de unas encuestas realizadas por la Casa de Velázquez en Cazalla de la Sierra (Sierra Norte de Sevilla) ponen de manifiesto que más de la mitad de las explotaciones mayores de 500 has existentes en el municipio surgieron entre 1970 y 1985, y que muchas de ellas cambiaron de propietario en este periodo. 
petencia de las producciones industriales y por la entrada desde Portugal de la peste porcina africana.

Las modificaciones introducidas en los sistemas de explotación ganadera se resumen en un cruce -cuando no sustitución- de razas autóctonas con otras importadas. Ello se traduce en una adulteración de su pureza genética, en una pérdida de la complementariedad ecológica entre las distintas especies que había caracterizado a las dehesas tradicionales y, sobre todo, en una fuerte dependencia del exterior por parte de unos animales poco adaptados al medio y necesitados de los piensos concentrados en su dieta alimenticia.

Con todo, el hecho más destacable es el abandono de que fue objeto la ganadería extensiva tradicional, cuyas producciones no podian competir ni en cantidad ni en periodicidad de abastecimiento con las ofertadas por las ganaderías industriales que se estaban implantando con el apoyo de la Administración- en los espacios llanos y mejor comunicados.

Estas mutaciones de los elementos configuradores de las dehesas tradicionales también han supuesto una reducción considerable de su superficie. Asi, a pesar de su escasa fertilidad edáfica, se calcula que durante los últimos treinta años han sido roturadas más de un millón de hectáreas de encinas y alcornoques adehesados, con la consiguiente acentuación de los procesos erosivos. Las dehesas que persisten han padecido un grave deterioro o tienden a convertirse en dehesas fósiles, en función de su intensificación productiva y de la falta de regeneración de su arbolado.

Las respuestas dadas ante la crisis por parte de las explotaciones serranas son muy diferentes en función de innumerables factores, entre los que destacan las situaciones socioeconómicas de partida y sus dimensiones territoriales.

Las grandes explotaciones son las que mejor se mantienen debido a que sus propietarios no viven de las mismas, pero a menudo modifican sus aprovechamientos convirtiéndose en cotos de caza y siendo repobladas con especies forestales ajenas a este agrosistema (eucaliptos y pinos). $\mathrm{Su}$ crisis se manifiesta igualmente cuando sus propietarios optan por continuar con los aprovechamientos anteriores, ya que suele mermar la superficie pastable por el avance del matorral y por el abandono de los cultivos; se descuida la vegetación arbórea; retroceden las razas autóctonas y se semi-intensifica la ganadería... 
Los pequeños propietarios y campesinos sin tierra, para quienes las dehesas constituian una forma de vida, se ven, por el contrario, obligados a emigrar. Ello provoca un abandono generalizado de las pequeñas explotaciones características de los ruedos de los pueblos, una mayor concentración de la estructura de la propiedad y la llegada de nuevos propietarios poco interesados en perpetuar un sistema que por otra parte desconocen.

\section{CuAdro I}

\section{ESTRUCTURA DE LAS EXPLOTACIONES DE SIERRA MORENA Y} RESPUESTAS ANTE LA CRISIS

\begin{tabular}{|c|c|c|c|c|c|}
\hline & $\begin{array}{l}\text { Usos } \\
\text { del } \\
\text { suelo }\end{array}$ & $\begin{array}{c}\text { Sistemas } \\
\text { ganaderos }\end{array}$ & $\begin{array}{c}\text { Tipo } \\
\text { de ganado }\end{array}$ & $\begin{array}{l}\text { Respuestas } \\
\text { ante la crisis }\end{array}$ & $\begin{array}{l}\text { Grupo } \\
\text { social }\end{array}$ \\
\hline $\begin{array}{l}\text { Gran } \\
\text { exploltación }\end{array}$ & $\begin{array}{l}\text { Dehesa } \\
\text { Matorral } \\
\text { Pastos } \\
\text { Cereal }\end{array}$ & Extensivos & $\begin{array}{l}\begin{array}{l}\text { Hetero- } \\
\text { geneidad: }\end{array} \\
\text { Porcino } \\
\text { Ovino } \\
\text { Vacuno } \\
\text { Caprino }\end{array}$ & 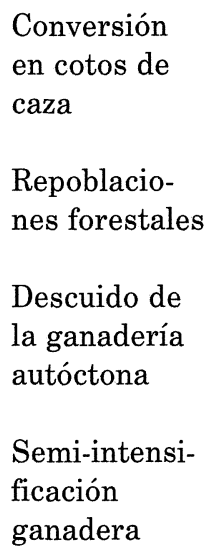 & $\begin{array}{l}\text { Profesio- } \\
\text { nales } \\
\text { liberales } \\
\text { Grandes } \\
\text { Terrate- } \\
\text { nientes }\end{array}$ \\
\hline $\begin{array}{l}\text { Pequeña } \\
\text { explotación }\end{array}$ & $\begin{array}{l}\text { Olivar } \\
\text { Huertos } \\
\text { Forrajes } \\
\text { Cereal }\end{array}$ & Intensivos & $\begin{array}{l}\text { Ganadería } \\
\text { para } \\
\text { autoconsumo }\end{array}$ & $\begin{array}{l}\text { Emigración y } \\
\text { desaparición } \\
\text { de } \\
\text { explotaciones } \\
\text { Reconversión } \\
\text { hacia el } \\
\text { vacuno de } \\
\text { leche ( un } \\
\text { hecho } \\
\text { puntual) }\end{array}$ & $\begin{array}{l}\text { Pequeños } \\
\text { agricultores } \\
\text { Jornaleros }\end{array}$ \\
\hline
\end{tabular}


El retrato resultante de este proceso es un territorio despoblado, en manos de grandes propietarios que no viven en él y que se desentendieron de la marcha de sus explotaciones en cuanto éstas dejaron de depararles beneficios económicos. Mientras tanto, empiezan a surgir intentos puntuales de salida de la crisis (introducción del vacuno de leche en la comarca cordobesa de Los Pedroches y apuesta por la transformación del porcino ibérico en la Sierra de Huelva), con dificultades de adaptación a la situación actual. Con todo, el desenlace final ha sido la quiebra de un agrosistema que tradicionalmente había demostrado saber mantener un equilibrio estable entre el hombre y el medio gracias a la explotación sincrónica de los usos agrícolas, ganaderos y forestales en una misma unidad de gestión.

A modo de paradoja con la situación arriba descrita, es en este contexto cuando se empieza a valorar desde instancias gubernamentales la importante función medioambiental y paisajística que este agrosistema desempeña. Es decir, la pérdida del valor económico de estos espacios, ya reducidos a la condición de marginales, ha sido la que ha dado origen a su percepción como paisajes de alto valor ecológico. Ello, encuadrado en una ideología clorofilica surgida entre la clase media urbana, ha llevado a su declaración oficial como espacios protegidos y a una gestión de los mismos por parte de la Administración pública que, pese a las intenciones teóricas de principio, contiene un sesgo proteccionista en el que los componentes del territorio producto de la acción humana no aparecen valorados en los términos que debieran.

\section{Elementos de las dehesas y politicas agroambientales}

La crisis de los aprovechamientos tradicionales de las áreas de dehesas no fue la resultante de una dinámica interna, sino que surgió como efecto de un cambio de concepción impuesto desde fuera. El modelo productivista de los años sesenta, con su dogma de crecimiento económico, provocó la homogeneización de territorios diferentes y la marginación de las áreas serranas. El resultado fue, según se ha visto, una mutación de los elementos configuradores de las dehesas tradicionales y la quiebra de este agrosistema.

El contexto económico actual (de problemas generados por los 
excedentes productivos, de creciente concienciación sobre la necesidad de respetar el medio ambiente y conservar el paisaje, de interés por revitalizar la economía de las áreas marginales, de apuesta por la corrección de los desequilibrios interterritoriales, etc.) está conduciendo a una nueva concepción con importantes repercusiones en los espacios situados al margen del esquema desarrollista. El productivismo a ultranza deja paso a una mentalidad agroambientalista que aboga por la recuperación de los agrosistemas tradicionales no productores de excedentes, a los que se asigna un papel fundamental en la corrección de las disfuncionalidades generadas por el modelo anterior: desequilibrios territoriales y deterioro del medio ambiente y el paisaje. Asi, las áreas marginales -entre ellas las dehesas- se convierten -en muchos casos de forma indirecta- en el punto de mira de las politicas agroambientales comunitarias. Pero tales politicas se encuadran asimismo en un paradigma exógeno a este ámbito mediterráneo y sureño que, en cierta medida, también pretende homogeneizar lo diferente al englobar bajo un mismo patrón -el que prevalece en el Norte de Europa- al conjunto de las áreas marginales. El resultado es una falta de aplicabilidad de los programas, cuando no una inadecuación entre los objetivos declarados y los resultados conseguidos que puede acarrear consecuencias no deseadas e irreversibles.

Esta nueva situación puede constituir, a pesar de lo anterior, una baza nada despreciable en favor de la recuperación de las dehesas. Pero hemos de tener en cuenta que la consecución de este objetivo debe pasar por una actuación simultánea sobre el conjunto de sus componentes. Es decir, los programas actuales sólo incidirán en la recuperación de este agrosistema en la medida en que sean capaces de encontrar una nueva situación de equilibrio, enmarcada en un modelo alternativo que sustituya al que sustentaba a las dehesas tradicionales. Pero estos programas no han sido diseñados en la mayor parte de los casos pensando en la recuperación de las dehesas; de ahí que no pasen de incidir de forma aislada en cada uno de sus componentes, sin tener en cuenta las repercusiones sobre el conjunto. Se echa, pues, en falta, una estrategia globalizadora que contemple el complejo sistema de interrelaciones característico de este agrosistema.

Con este punto de vista se presentan ahora algunas disfuncionalidades y resultados no previstos de las politicas vigentes en los prin- 
cipales elementos que conforman las dehesas: la ganadería, el espacio forestal, el capital humano y el paisaje.

Los programas con incidencia en la ganadería.-La ganadería extensiva constituye, según se ha señalado más arriba, un elemento consustancial del agrosistema dehesas. Se trata, en términos generales, de una ganadería ubicada en grandes propiedades (de más de 500 hectáreas), basada en la explotación de razas autóctonas (retinta y ganado de lidia, en el bovino; merina en el ovino, serrana en el caprino; ibérica en el porcino), con distintos niveles de cruces con otras importadas; pero, en todo caso, poco mejoradas desde el punto de vista genético y con graves problemas productivos y comerciales.

La reciente apuesta por la extensificación productiva por parte de la politica agraria europea,y la modulación de las ayudas ganaderas en función de la superficie pastable, ha situado a Sierra Morena en una de las pocas zonas andaluzas que se ajusta al patrón de 2 $\mathrm{UGM} / \mathrm{Ha}$ de superficie forrajera requerida para el acceso a las primas comunitarias. Ello supone que una explotación de dehesas con 500 hectáreas -que puede dar cabida a unas 50 vacas nodrizas, a 200 ovejas y a unas 100 cabras- puede estar percibiendo en torno a diez millones de pesetas anuales en concepto de primas. Con ello, las grandes propiedades de Sierra Morena vuelven a ser «rentables». Pero se trata de una rentabilidad hasta cierto punto ficticia, amparada en las subvenciones y no en una mejora de las condiciones de explotación.

Por otra parte, hay que tener en cuenta que las ayudas están recayendo en unos propietarios calificados acertadamente de absentistas (Roux, 1975-1981, y Casa de Velázquez, 1986), con elevados niveles de ingresos, para quienes la actividad ganadera constituye poco más que un pasatiempo. En resumidas cuentas, las primas ganaderas - que pretenden ser ayudas al complemento de renta- están en muchos casos incidiendo en el colectivo menos necesitado. Asimismo, pueden estar disuadiendo a los ganaderos de la introducción de las mejoras productivas que sus explotaciones necesitan, convirtiendo a la ganadería extensiva de las dehesas en una ganadería subsidiada e hipotecando su futuro en función del devenir de las ayudas.

En el otro extremo, los programas actuales están imponiendo un cierto freno -bien por penalizaciones directas, bien por omisión de ayudas- a los escasos intentos de salida de la crisis. Un ejemplo de

$$
-214-
$$


ello lo constituye el vacuno de leche de la comarca cordobesa de Los Pedroches, introducido a partir de los años sesenta ante la creciente demanda de leche de vaca por parte de una población en ascenso que estaba incrementando su nivel de vida. La nueva actividad, que contó en sus inicios con el apoyo de la Administración -vía subvención de créditos-, significó una alternativa de futuro para las pequeñas explotaciones familiares cuyos miembros hablan quedado sin trabajo ante la crisis de las dehesas, y que de otra forma hubiesen desaparecido. El éxito de esta empresa -modernización del sector, reestructuración de las explotaciones, incremento de los rendimientos (unas medias superiores a 7.000 litros/vaca/año y más del $80 \%$ de leche tipo A)- no se ajusta a los recortes impuestos por la Comunidad a la producción lechera, y los pequeños ganaderos de Los Pedroches -que aún no han amortizado los créditos contraídos- deben pagar las consecuencias de haber optado por el incremento de la productividad, que por otra parte era lo que se les pedía. Es decir, la política de recorte de excedentes -a veces enmascarada con principios agroambientalistas- puede suponer un freno para las escasas expectativas de dinamismo surgidas en las áreas marginales, como está ocurriendo en Pedroches. Así, aunque la opción por el vacuno de leche adoptada por esta comarca cordobesa no está exenta de problemas -como su excesiva dependencia de los concentrados estadounidenses- hay que reconocerle, como mérito propio, el de haber conseguido retener la población en un espacio marginal y, sobre todo, involucrar a los ganaderos en el proceso de transformación y comercialización del producto gracias a su inclusión en una importante cooperativa (la Cooperativa del Valle de los Pedroches-COVAP); dos hechos, por otra parte, que resultan excepcionales en Andalucía.

El segundo de los ejemplos de apuesta por la competitividad -la transformación y comercialización de los productos del cerdo ibérico- tampoco está exento de problemas, no tanto en este caso por penalizaciones directas como por la omisión de ayudas. Si bien las empresas dedicadas al sector (entre las que destacan las situadas en Jabugo, en la Sierra de Huelva) están recibiendo significativos apoyos para la mejora de sus instalaciones, los ganaderos están al margen de todo tipo de ayudas por explotar una especie -el porcino- que no se ajusta a los canones de lo que la Comunidad considera como ganadería extensiva. Con la vista puesta en las grandes explotaciones 
industriales de Alemania, Holanda o Bélgica, no se puede pensar que exista un porcino extensivo -el cerdo ibérico- en las dehesas de Sierra Morena, ni que éste constituya un elemento fundamental en la configuración de un agrosistema que se pretende recuperar por otras vías.

Los programas con incidencia en las especies forestales.Las especies forestales (encinas y alcornoques) constituyen un elemento de primer orden en el agrosistema dehesas, que no es otra cosa que un «monte aclarado» o «monte hueco». Además de proporcionar la principal fuente de alimentación del ganado (las bellotas), la masa arbórea resulta fundamental para garantizar el crecimiento de los pastos y la maduración de los cultivos, que no podrian desarrollarse sin la sombra de los árboles.

Pero ya se ha visto como el incremento salarial llevó a los propietarios de las dehesas a descuidar esta vegetación, con consecuencias nefastas para el conjunto del agrosistema. Posteriommente, los reiterados intentos por parte de la Administración de recuperar las masas de encinas y alcornoques no han dado los resultados previstos. Así por ejemplo, si bien el Plan Forestal Andaluz (1989) preveía una plantación media anual de 10.000 ha de quercíneas en sus sesenta años de vigencia, a costa de las superficies invadidas por el matorral, los resultados del periodo 1988-1992 distan mucho de aproximarse a estas previsiones: un arranque de cerca de 19.000 ha de encinas y un avance del alcornoque por apenas 2.000 ha (Junta de Andalucía, 1994). Otro tanto cabe señalar en relación a la Línea de Fomento de Encinares y Alcornocales, incorporada en 1989 (Orden de la Consejería de Agricultura y Pesca de 7 de abril de 1989) al Programa Nacional de Fomento de las Inversiones Forestales en Montes Privados que venía funcionando desde 1969, y que sólo ha supuesto la plantación de 182 ha de estas especies entre 1990 y 1993 en toda Andalucía.

La escasez de presupuesto para afrontar la ejecución del Plan Forestal y la necesidad de contar con la participación financiera de los propietarios (que deben sufragar entre el $40 \%$ y el $50 \%$ de los gastos) en la Línea de Fomento de Encinares y Alcornocales en Montes Privados, se han conjugado para propiciar estos pobres resultados. Pero la situación está cambiando a raíz de la aplicación del nuevo programa comunitario de reforestación, que además de hacerse cargo de la 
totalidad de los gastos, ha incrementado considerablemente la cuantía de las subvenciones.

Este programa contempla tres tipos de primas, de cuantía variable según la especie por la que se opte en la reforestación: una prima de reforestacion, percibida una vez realizada la plantación, que asciende a 325.000 pesetas/ha si se opta por las quercíneas; una prima de mantenimiento de la superficie forestada, que se percibe durante cinco años, y que en las reforestaciones con quercíneas asciende a 30.000 pesetas/ha; y una prima compensatoria, con una vigencia de veinte años, y un importe de 35.000 pesetas/ha para encinas y alcornoques. Así, según nuestros cálculos, una explotación de dehesa que destine 25 ha a la reforestación con estas especies puede llegar a percibir 28.750.000 pesetas en los veinte años que aplique el programa. No es, pues, de extrañar, que este programa haya supuesto, en sus dos primeros años de vigencia (1993-1994), la plantación de cerca de 30.500 ha de encinas y alcornoques en el conjunto de Andalucía (cerca del 65\% de la superficie afectada por el mismo), en su mayor parte situadas en Sierra Morena.

Las objeciones que cabe realizar en relación a las implicaciones futuras de este programa son muy numerosas, como ya señalábamos en otro trabajo (Silva, 1995). Así por jemplo, la encina requiere un cuidado constante, que sobrepasa ampliamente los cinco años de percepción de la prima de mantenimiento habilitada para tal fin. $\mathrm{Y}$ el descuido de esta vegetación por parte de los propietarios de las dehesas en cuanto cesaron los benefcios económicos, nos inclina a pensar en un abandono de este mantenimiento una vez que la ayuda se extinga. Por otra parte, la regeneración de esta masa forestal en superficies degradadas como la que nos ocupa no está en absoluto garantizada. En este sentido, el Plan Forestal Andaluz recomienda inciar la reforestación con la «etapa de pinares» -a los que, por su resistencia a la sequía, se considera especialmente aptos para aguantar una insolación directa en los primeros años de desarrollo de la planta- a modo de antesala de las repoblaciones con encinas. Pero los propietarios particulares se han inclinado por reforestar directamente con quercineas movidos por las ayudas más cuantiosas que éstas reciben. Con todo, el hecho más preocupante en relación con la aplicación de esta medida se refiere a la nueva recolonización de que pueden estar siendo objeto las dehesas -como ya se empieza a detectar-, con la llegada de nuevos propietarios alentados por 
los beneficios monetarios deparados por un programa cuyas ayudas superan en muchos casos al importe de la tierra (Ojeda, J., y Silva, R., 1996).

Los programas con incidencia en el elemento humano.-Las dehesas no constituyen un ecosistema natural, sino cultural, de ahí que el proceso emigratorio se erija en una de las principales causas desencadenantes de su crisis.

El cierre de la válvula de escape que supuso el empleo en otros sectores, la congestión de las áreas urbanas y la migración de retorno -en el contexto de la filosofía agroambientalista hoy dominante- ha llevado a reconocer, a efectos gubernamentales, la necesidad de retener población en las áreas marginales. Para ello se habilitan una serie de ayudas dirigidas, bien al mantenimiento de la agricultura -a la que se empieza a considerar como un elemento más del paisaje-, bien al ensayo de nuevas formas de ganarse la vida, eso sí, fundamentadas en el aprovechamiento de los recursos propios.

La misma Comisión Europea reconoce que "es necesario mantener un número suficiente de agricultores en la tierra. Es la única forma de preservar el medio ambiente, un paisaje milenario y un modelo de agricultura que es expresión de un modelo de sociedad» (Comisión de la CE, 1991). Pero en las áreas de dehesas -muy castigadas por la emigración y por la desaparición de explotaciones-, no basta con mantener población asociada a la tierra, sino que hay que atraerla; sobre todo si se tiene en cuenta que la emigración afectó a los más jóvenes, y que el envejecimiento que presentan los agricultores «de siempre»-los que se quedaron- se constituye en un handicap para el relevo generacional. Por otra parte, resulta impensable que los antiguos emigrantes -y aún menos sus hijos- retomen una actividad escasamente remunerada, desarrollada en un medio físico hostil, con crecientes limitaciones legislativas -a veces un tanto ridículas- relacionadas con su declaración como espacios protegidos.

Desde la perspectiva de la Europa dominante -con un amplio tejido de explotaciones familiares- quizás se conciba que las indemnizaciones compensatorias para áreas desfavorecidas pueden contribuir a paliar las limitaciones antedichas. Pero en las áreas de dehesa este tipo de explotaciones hace mucho que desaparecieron ${ }^{2}$, por lo que

\footnotetext{
${ }^{2}$ Una excepción sería la comarca de Los Pedroches, pero, según se ha visto, la opción por el vacuno de leche que permitió su persistencia tiene comprometido el futuro a raíz de la aplicación de las cuotas lecheras.
} 
estas ayudas -por otra parte muy escasas- están recayendo igualmente en las grandes explotaciones, cuyos titulares pueden ser calificados de cualquier cosa, excepto de agricultores.

En otro orden de cosas, teniendo en cuenta que los pobladores de las áreas marginales no pueden vivir sólo de la agricultura, se inician una serie de programas que intentan conseguir una cierta diversificación económica. En este contexto se encuadra la Iniciativa LEADER que, como es sabido, pretende experimentar nuevas estrategias de creación de empleo a base del aprovechamiento de los recursos endógenos. Pero en la lectura que desde las áreas de dehesa se ha hecho del programa, no queda claro qué significa eso de «endógeno»-si es que este término puede ser definido a un nivel más general-: ¿se refiere a los recursos físicos?, ¿al paisaje en su conjunto?, ¿a alguno de sus componentes?, ¿a las formas tradicionales de ganarse la vida?, $\iota^{\mathrm{a}}$ la población de siempre?

A tenor de la aplicación del LEADER I en Sierra Morena, parece que lo endógeno es el paisaje; al que, por otra parte, se considera como algo dado, sin plantearse actuar sobre él en un intento de corregir la degeneración de que éste ha sido objeto. Así, en el primer periodo de vigencia de esta iniciativa (1991-1993) han estado funcionando en Sierra Morena dos proyectos LEADER: el de la Sierra Norte de Sevilla y el de Riotinto (en el Andévalo onubense). Ambos han centrado su estrategia de diversificación económica en la promoción de un turismo rural (con más del 70\% del presupuesto en la comarca sevillana y en torno al $77 \%$ en la onubense) que se pretende apoyar en la «venta» del paisaje de las dehesas, aunque a ello se añada la rehabilitación turística de unas antiguas instalaciones mineras, en el caso de Riotinto. Es decir, parece que se está optando por pasar de una «monoespecialización agraria» a un «monocultivo turístico»; por lo que se trataría -a modo de paradoja con la filosofía del programa-, más que de diversificar la economía, de cambiar de sector dominante. Pero el problema más grave, según nuestro punto de vista, se refiere a quiénes son los interlocutores encargados de la ejecución de ambos programas. Desde instancias comunitarias se propone que esta tarea recaiga en los agentes locales; pero en territorios como en el que nos ocupa -donde la iniciativa empresarial es mínima- estos agentes se identifican con los principales grupos de presión, con intereses muy distantes de los que mueven al conjunto de la población. 
Ello se ha traducido en una escasa identifcación popular con el programa; máxime si se tiene en cuenta el descuido de que han sido objeto los sectores tradicionales y, en especial, determinadas industrias agroalimentarias de carácter artesanal con un fuerte arraigo en la zona-fabricación de anisetes, industrias chacineras...

Con todo, la oportunidad de recibir fondos comunitarios $-\mathrm{y}$ el ejemplo de las cuantiosas inversiones asociadas al LEADER I: 9.998 millones de ECUs en la Sierra Norte de Sevilla y 1.669 millones en Riotinto-, ha llevado a que todas las comarcas de Sierra Morena apuesten por esta iniciativa. Así, en la segunda fase de aplicación de este programa (1994-1999) se han aprobado los siguientes LEADER II para la zona: Sociedad de Desarrollo de Sierra Morena Cordobesa, Los Pedroches de la Provincia de Córdoba, Iniciativa LEADER, Sierra de Aracena y Picos de Aroche de Huelva, Asociación para el Desarrollo Rural del Andévalo Occidental de la Provincia de Huelva, CEDER de la Cuenca Minera de la Provincia de Huelva (antiguo LEADER de Riotinto), Asociación para el Desarrollo del Corredor de la Plata en la Provincia de Sevilla y Ecodesarrollo de Sierra Morena en la Sierra Norte de Sevilla.

Todos los proyectos arriba relacionados han quedado incluidos en la modalidad de Adquisición de Capacidades, incluso los que ya habían funcionado en el periodo de vigencia del LEADER I ${ }^{3}$. Por lo tanto, aún se desconoce qué sectores se constituirán en sus protagonistas o quiénes serán sus interlocutores. En cualquier caso, la oportunidad de recibir subvenciones puede atraer a nuevos especuladores a un territorio, ya de por sí amenazado -desde varios frentes- y cimentar el proceso de «desamortización encubierta» que viene padeciendo desde hace años.

El paisaje.-Entendiendo el paisaje de dehesa como el resultado formal de la conjunción de sus componentes, resulta interesante incluirlo en este análisis por la gran importancia que se le está confiriendo en la actualidad. Parte de las estrategias de superación de la-

3 El LEADER II estructura los CEDERs en tres categorías: Adquisición de Capacidades, Programas de Innovación Rural y Programas de Cooperación Trasnacional -se refiere a conciertos de cooperación entre grupos pertenecientes a más de un Estado. La modalidad de adquisición de capacidades comprende una serie de ayudas de asistencia técnica -previas a las inversiones específicas- dirigidas a la realización de estudios y diagnósticos del territorio en cuestión, a la constitución del grupo de acción local, a la búsqueda de financiación y a la sensibilización y movilización de la población. 
crisis serrana pasa por la recuperación, protección y conservación de sus paisajes.

Tras la asunción de competencias por parte de la Administración autónoma andalaza se elaboran una serie de estudios y programas (Planes Especiales de Protección del Medio Físico, programas que desarrollan la Ley 25/1982 de Agricultura de Montaña...) que ponen en evidencia los valores ambientales de ciertos paisajes y el peligro de pérdida de los mismos que un desarrollo productivista podría suponer. Esta constatación lleva a la Administración regional a la aprobación de una Ley de Inventario de Espacios Protegidos Andaluces (Ley 2/1989), que supone la declaración de cuatro Parques Naturales en Sierra Morena (Sierra de Cerdeña y Montoro, Sierra de Hornachuelos, Sierra Norte de Sevilla y Sierra de Aracena y Picos de Aroche), con una superficie conjunta de 457.254 has, en torno a la cuarta parte de todo su territorio.

La figura de Parque Natural en dicha Ley (basada en la Ley estatal 4/1989 de Conservación de los Espacios Naturales y de la Flora y la Fauna Silvestres) tiene su modelo en el Parque Natural Regional francés, pero difiere de aquél tanto en su inserción normativa (Código Rural francés/Ley Ambiental española), como en el procedimiento y la iniciativa de su declaración (contrato social o Carta del Parque, en el caso francés, y declaración administrativa en el español) y en el posterior desarrollo de su gestión (Gómez Mendoza, J., 1995).

La responsabilidad de gobiemo parece que conduce a una desvirtuación del modelo adoptado, en la medida en que se abusa de la visión medioambientalista en detrimento de la rural, y no se cuenta con la población autóctona a la hora de declarar protegidos sus propios espacios. Esta visión administrativa de la realidad resulta, a la postre, superficialmente globalizadora, ya que no conoce o no puede tener en cuenta el funcionamiento interno de los sistemas y las interrelaciones de todos y cada uno de sus componentes, en definitiva, los vectores que dan vida propia a cada territorio.

Si se piensa por un momento en lo que eran las dehesas tradicionales, tal como han sido descritas, se comprobará que la sabiduría que las sostenía se basaba en un profundo conocimiento de cada componente, en un manejo adecuado de los mismos en función de sus tiempos y sus complementariedades y en un aprovechamiento in- 
tegral. Pero al parecer se está perdiendo esta perspectiva histórica, en un intento de recuperar la morfología del conjunto, aun a costa de la desatención de sus partes. Como consecuencia de ello, los habitantes de estas zonas no se encuentran identificados con lo que -desde la administración- se pretende que sea su territorio y se convierten en interlocutores pasivos de una situación que soportan -por los benefcios que les depara-, pero que en absoluto comparten.

En el fondo de esta situación subyace la falta de distinción entre lo que es una dehesa -entendida como un sistema vivo de explotación rural- y una superficie adehesada. Muy ilustrativo de ello resulta la confusión entre dehesa y bosque de quercíneas que se recoge en El Plan de Ordenación de los Recursos Naturales de la Sierra de Aracena y Picos de Aroche en el que se observan las siguientes connotaciones:

«Se entiende por dehesas y bosques de quercíneas las manchas de vegetación constituidas por encinares, alcornocales, quejigales, castañares o robledales, ya se presenten como formaciones monoespecíficas o mezclados, y con porte arbustivo o arbóreo» (art. 173 P.O.R.N. del Parque Natural de la Sierra de Aracena y Picos de Aroche). En el capítulo de aquel documento relativo a la ordenación de los recursos en el interior del parque se presta una especial atención a los recursos naturales (suelo, formas estructurales, vegetación, fauna) al tiempo que se infravaloran aquellos componentes resultantes de la intervención humana. Así por ejemplo, las directrices que regulan la gestión ganadera se refieren sobre todo a la consideración de esta actividad como una amenaza para el desarrollo de la vegetación, y no como un elemento consustancial al mantenimiento de las dehesas y de su propia biodiversidad (Parra, F., 1990).

Otro tanto cabe señalar en relación a los recursos forestales, en cuya gestión se desestima la intervención del hombre, por lo que no resultan excepcionales aseveraciones como la siguiente: «los montes como ecosistemas forestales [se refiere a las dehesas] deberán ser gestionados de forma integrada, contemplándose, conjuntamente, la vegetación, la fauna y el medio físico que los constituyen, con el fin de conseguir un aprovechamiento sostenido de los recursos naturales...» (art. 62 del citado P.O.R.N.).

Evidentemente, esta pretendida gestión integrada y esta sostenibilidad de los recursos pasa por alto el papel desempeñado por el 
hombre (con sus actividades) en la configuración del paisaje de las dehesas. La percepción sesgadamente ambientalista -vinculada quizás a la figura de Parque Natural como producto original de colonias de poblamiento anglosajonas, donde hay más tierra que gente y no se cuenta con monumentos históricos- incapacita a los propios administradores y gestores de los Parques Naturales ${ }^{4}$ para entender que el territorio de estas áreas de vieja civilización es un producto cultural $\mathrm{y}$, consecuentemente, sus paisajes son eminentemente rurales.

Esta forma de entender las cosas nos lleva finalmente a señalar una nueva imprecisión: aguélla que subyace tras el enarbolado concepto de desarrollo sostenible en el que se pretende apoyar la gestión de tales espacios. A las preguntas ya tópicas de si el desarrollo puede ser o no sostenible, de qué debe ser sostenido o de hasta dónde las posibilidades prácticas de sostenibilidad, quizás podría responderse con una frase ingeniosa: «A lo mejor, si nos ponemos a profundizar, llegamos al acuerdo de que el único recurso que hay que cuidar es a la gente» (Caballero Ruano, 1995).

\section{Consideraciones finales}

La dehesa ha demostrado históricamente ser un sistema especialmente adecuado para explotación de los recursos agrobiológicos existentes en la montaña media mediterránea (mantiene el equilibrio producción/recursos, crea un paisaje singular, genera puestos de trabajo...), de ahí que se reclame su recuperación. Para ello debemos volver la mirada a lo que eran las dehesas tradicionales, pero no para reproducirlas, sino para intentar adaptarlas a la situación actual prescindiendo de determinados condicionantes socio-económicos que permitieron su estructuración en el pasado: economía autárquica, existencia de una mano de obra mal pagada... La demostrada flexibilidad del agrosistema ha ido permitiendo su adaptación a nuevas tecnologías, siempre que se respetasen los principios básicos de su configuración, organización y funcionamiento.

Según nuestro punto de vista, no se puede pensar en recuperar

${ }^{4}$ Los máximos responsables de la gestión de los Parques Naturales son denominados, como en los Parques Nacionales, directores-conservadores. 
las dehesas sin dar pruebas de su utilidad económica. Pero ello requiere una planificación adecuada de todos los recursos susceptibles de generar renta y empleo, así como la búsqueda de un mercado alternativo para sus producciones amparado en un distintivo de calidad. El contexto socio-econonómico actual se constituye en una baza nada despreciable para intentarlo, pero -a tenor de los resultados de la aplicación de algunos programas- no tanto para conseguirlo. Mientras tanto, el producto de la aplicación de las nuevas medidas está provocando dos efectos que resultan contraproducentes: la configuración de una región subsidiada y, en íntima relación con ello, la consolidación de los procesos de neocolonialismo y desamortización encubierta que viene padeciendo Sierra Morena desde hace varias décadas.

Si se tiene en cuenta que la población que ha quedado allí es poco numerosa y especialmente culta en relación con su territorio (Mora Galiana, J., 1996), que sus expectativas económicas no están aún sobredimensionadas, y que el medio físico no resulta de difícil manejo en esta montaña media mediterránea de cuarcitas, pizarras y granitos, habría que arriesgarse a efectuar una propuesta que apoye la autogestión inmediata del espacio y de los paisajes en que vive.

\section{BIBLIOGRAFÍA}

Caballero Ruano, M.: Prólogo del libro de Wladimiro Rodríguez El agua en Canarias y el siglo XXI. Cabildo Insular de Gran Canaria, 1995.

Casa de Velázquez (1986): Supervivencia de la Sierra Norte de Sevilla. Evolución de los paisajes y ordenación del territorio en Andalucia Occidental. Ministerio de Agricultura, Pesca y Alimentación, Junta de Andalucía y Casa de Velázquez.

Comisión de las Comunidades Europeas (1991): Evolución y futuro de la PAC. Bruselas, COM (91) final.

Decreto 98/1994, del 3 de mayo, por el que se aprueba el Plan de Ordenación de los Recursos Naurales y el Plan Gestor de Uso y Gestión del Parque Natural Sierra de Aracena y Picos de Aroche. BOJA núm. 98, de 30 de junio de 1994.

Gómez Gutiérrez, J. M. (1987): «El monte adehesado: situación económica y ecológica actual», Revista de Estudios Agrosociales, núm. 142, pp. 171-193.

Gómez Mendoza, J. (1995): «Desarrollo rural y espacios naturales protegidos», en RA. mos, E., y Cruz, J.: Hacia un nuevo sistema rural. Ministerio de Agricultura, Pesca y Alimentación. Madrid, pp. 381-410.

IBÁÑEZ, J. J., y otros (1981): «Propuesta de ordenamiento de los agrosistemas de dehesas en la península ibérica», Revista de Estudios Agro-Sociales, núm. 114, pp 39-79.

Junta de Andalucía (1989): Plan Forestal Andaluz. Consejería de Agricultura y Pesca, Instituto Andaluz de Reforma Agraria y Agencia de Medio Ambiente. 
- (1994): Anuario de estadísticas agrarias y pesqueras. Andalucía, 1992. Consejería de Agricultura y Pesca.

- (1995): La Agricultura y la pesca en Andalucia. Memoria 1994. Consejería de Agricultura y Pesca.

Mora Galiana, J. (1996): «Sentir y comprender la realidad de la Sierra». III Encuentro de Escritores de la Sierra. Galaroza. Huelva. Ejemplar mecanografiado.

OJEDA, J., y SILVA, R. (1996): «Sierra Morena, una montaña media andaluza ante el reto europeo», Montagnes Méditerranénnes. Université Joseph Fourier. Grenoble (en prensa).

Parejo Delgado, C. (1995): El medio rural en Andalucia. Editorial Librería Agora. Málaga.

PARRA, F. (1990): «La política de Espacios Naturales: una historia ambigua», Ciadad y Territorio, núm. $>83-1$.

Roux, B. (1975): La crisis agraria de la sierra andalaza. Un estudio económico de las empresas ganaderas de la provincia de Huelva. Instituto de Desarrollo Regional. Universidad de Sevilla.

Roux B. (1981): «Ganadería y ganaderos en el área de Sierra Morena», Revista de Estudios Agro-Sociales, núm. 116, pp. 264-270.

Silva PÉRez, R. (1995): «De la deforestación a la reconversión de terrenos agrícolas en forestales. Un análisis crítico», en Cambios regionales a finales del S. XX. Actas del XIV Congreso Nacional de Geografía. Salamanca, pp. 205-209.

Valle Buenestado, B. (1985): Geografía Agraria de los Pedroches. Diputación Provincial. Córdoba.

- (1995): «Propiedad y actividad agraria en espacios naturales protegidos», en Propiedad, actividad agraria y medio ambiente en España y América Latina. Servicio de Publicaciones de la Universidad de Córdoba. Córdoba, pp. 109-145.

RESUMEN: Este artículo es una reflexión sobre las aplicaciones de las políticas agroambientales comunitarias en las dehesas de Sierra Morena. Tras la presentación de los principios reguladores de las dehesas tradicionales y el análisis de su desarticulación en la etapa desarrollista, se intenta averiguar las posibilidades que ofrecen las políticas comunitarias para restablecer este agrosistema. La principal conclusión del estudio es que las medidas aplicadas no están produciendo el efecto positivo esperado. Los programas con una mayor incidencia (primas ganaderas, programa de reforestación, indemnizaciones compensatorias...) no han sido diseñados pensando en la recuperación de la dehesa, de haí que no pasen de incidir de manera aislada en cada uno de sus componentes, sin tener en cuenta las repercusiones sobre el conjunto. En definitiva se termina abogando por la búsqueda de una estrategia globalizadora para la recuperación de este agrosistema, cimentada en la secular sabiduría que tienen de este espacio y estos paisajes sus pobladores de siempre.

Palabras Claves. Medio ambiente, agricultura, ganadería, forestal, paisaje,dehesa, políticas.

ABSTRACT: This paper tries to think about the environmental and agricultura european policies applied to the montainous «dehesas» of Sierra Morena. After setting up the regulation principles of the traditional dehesas its breaking up after the economic development stage was analized. The opportunities of reequilibrium for this agrosystem making use of the european community policy were also investigated. The main conclusion of this study was that the european applied measures have not produced, so far, the expected positive effects. The programs that fall most heavily on this system (cattle aids, reforestation programs, compensating indemnifications...) have not been conceived for 
the dehesas recuperation as a whole. This is why the effect can only be noticiable separately on each element without looking at the repercussions over the system alltogether. Finally, positions are taken towards a wide sense strategy for this agrosystem recuperation based on the ancient wisdom of its inhabitants about this landscape and territory.

KEY WORDS. Enviroment, agriculture, cattle, forestry, landscape, dehesa, policy.

RÉSumÉ: Cet article est une réflexion sur les applications des politiques agro-environementales communautaires dans les «dehesas» de Sierra Morena. Après la presentation des principes régulateurs des «dehesas» traditionelles et l'analyse de sa desarticulation lors de l'étape de développemente (années 60 ), nous essayons de vérifier les possibilitésqu'offrent les politiques communautaires pour rétablir cet agrosystème. La pricipale conclusion de l'étude est que les mesures appliquées ne produisent pas l'effect attendu. Les programmes ayant le plus d'incidence (prime à l'élevage, programme de reforestation, indemnisations compensatoires...) n'ont pas été developpés en pensant à la récupération de la "dehesa» comme systèmes à part entière, ce qui ne veut pourtant pas dire qu'ils n'aient pas une influence isolée sur chacun de ses composants, mais ils n'ont pas de répercution sur l'ensemble. Enfin, nous terminons en plaidant pour la recherche d'une stratégie globalisante permettant la sauvegarde de cet agro-système, ancrée dans la culture ancestrale de ses populations et traduite dans l'espace et les paysages.

Mots CLEFS. Environement, agriculture, élevage, agro-silvopastoral, paysage, «dehesa» politiques. 